\title{
A viscoelastic - viscoplastic material model for superalloy applications
}

\author{
J. P. Rouse ${ }^{\text {a,* }}$, B. Engel ${ }^{\text {a }}$, C. J. Hyde ${ }^{\text {a }}$, S. J. Pattison ${ }^{\text {b }}$, M. T. Whittaker ${ }^{\text {, }}$ J. P. Jones ${ }^{\text {c }}$, B. Cockings ${ }^{\text {, }}$, \\ N. C. Barnard \\ ${ }^{a}$ Gas Turbine and Transmission Research Centre (G2TRC), University of Nottingham, United Kingdom. \\ ${ }^{b}$ Rolls-Royce plc., Derby, United Kingdom \\ ${ }^{c}$ College of Engineering, Swansea University, Swansea, United Kingdom
}

\begin{abstract}
An understanding of rate dependency over a wide range of time scales is vitally important in approximating the transient response of critical components operating in extreme environments. Many examples of viscoplastic model formulations can be found in the literature, wherein all rate dependency is assumed to occur after yielding. Such models neglect any viscous effects during elastic deformation. In the present work, a unified viscoelastic - viscoplastic material model is developed for the Nickel superalloy RR1000. Particular emphasis is placed on model parameter determination, which is accomplished using standard cyclic plasticity and stress relaxation experimental data.
\end{abstract}

Keywords: viscoelasticity, viscoplasticity, stress relaxation, RR1000, superalloy

\section{Introduction}

It is well known that material deformation behaviour is time dependent in many situations; particularly when said material is loaded at elevated temperature. For example, numerous experimental studies published in the literature have demonstrated that the stress states measured in metals held at constant strain will reduce with time through the accumulation of inelastic strain (that is to say, the component of strain which is not recovered on unloading). It is important to note that time dependencies are commonly observed over a large range of loading levels. It is easily shown that loading rates influence the apparent "elastic" modulus observed when metals are tested at elevated temperature and stresses may relax well below the apparent yield stress for the material. Note that the term elevated temperature here refers to temperatures where creep may be considered to be a relevant deformation mode, typically assumed to be approximately $40 \%$ of a material's melting temperature [1]. Despite these observations, it is common to see attempts to model the constitutive response of metals at high temperature using unified elastic viscoplastic assumptions. In these formulations all time dependency is assumed to take place during plastic deformation (i.e. once the stress state exceeds the yield condition). As such, stresses can only relax back to the value defined by the yield locus (which in most cases is an initial yield strength modified by a set of hardening related stress quantities). While it is possible to artificially make this type of model agree with experimental data by defining a very low initial yield stress this strategy is obviously flawed. By way of example, if a low yield stress value were to be adopted in a model the concept of strain decomposition would loose all meaning, suggesting that strain based fatigue lifing methods (such as those of Ince and Glinka [2]) could not be reliably applied. In the present work viscoelastic time dependencies, which are associated with rate dependent effects observed in what would normally be described as the elastic domain, are coupled with viscoplastic formulations by defining a secondary limiting surface that is enriched with its own flow rule.

${ }^{*}$ Corresponding author

URL: james.rouse@nott ingham.ac.uk (J. P. Rouse)

Preprint submitted to Elsevier 
The literature is rich with examples of viscoplastic models applied to Nickel superalloys. Many of these use concepts that were popularised in the work of Chaboche and Rousselier [3, 4]. Such models are much simpler than the multi-surface plastic models of Mroz [5] and Dafalias-Popov [6] and typically decompose total strain into elastic and viscoplastic components, with the definition of a flow rule in the latter to describe time dependent effects. By defining a yield function, which describes the location of the yield locus in the deviatoric stress space, loading responses can be judged as either elastic or elastic viscoplastic. Yield functions are typically formulated to evolve with a set of hardening parameters which quantify, for example, the change in the yield locus position or size due to kinematic or isotropic hardening, respectively. An excellent review of several of continuum plasticity theories can be found in the work of Chaboche [7]. Of particular note in so called Chaboche models are the wide range of flow rules that have been applied and the definition of multiple back stress components. Such kinematic hardening laws often build on the simple hardening modulus models of Prager [8] and Ziegler [9] to include dynamic recovery (as in the Armstrong-Frederick model [10]) effects and beyond. An isothermal application of a power law flow rule viscoplastic model may be found in Zhan et al. [11], wherein a Nickel superalloy tested at $650^{\circ} \mathrm{C}$ was considered and modelled with an enhanced kinematic hardening model that accounted for memory effects. Of particular note in this work was the inclusion of correlation coefficients for the material parameters required by the model. Such information is useful in performing simulations which consider how variance affects a predicted material response as sampling can be performed from "principal" components (note that these are linear combinations of the material parameters which give rise to a diagonal covariance matrix). The work of Cornet et al. [12] is a further example of a power law flow rule application for a viscoplastic material model. This work considered isothermally loaded $\left(650^{\circ} \mathrm{C}\right)$ RR1000 and modified hardening laws through the inclusion of a classical continuum damage parameter. The work of Zhao and Tong [13] is similar to that of Cornet et al., however damage terms are not considered and the main focus of the work is the application of a power law viscoplastic model to study local crack tip behaviour. Viscoplasticity has also been applied in finite strain formulations, as evidenced in the recent work by Kirka and Neu [14]. Here, crystal plasticity models were developed for the $\gamma$ and $\gamma^{\prime}$ phases that often form in Nickel superalloys. A combined power law and exponential flow rule was used to describe viscoplastic flow, with both isothermal and an-isothermal loading profiles considered. Similar work has been conducted by Zhang and Oskay [15] who also worked with finite strain formulations to describe a IN 617 material at $950^{\circ} \mathrm{C}$ using an exponential flow rule attributed to Busso.

Applications of combined viscoelastic and viscoplastic model formulations are less common in the literature. Several examples, including work by Yu et al. [16] and Launay et al. [17], use Kelvin-Voigt elements to model viscoelastic deformation in fibre reinforced composite materials. Standard flow rules are typically used to describe viscoplastic phenomena, with so called anhysteretic loading waveforms (wherein intermediate hold periods are introduced during loading) used to generate experimental data for parameter calibration. Similar methods were used in the work of Benaarbia et al. [18] for the description of P91 chromium steel at $600^{\circ} \mathrm{C}$. A related formulation was also used for P91 by Cai et al. [19] however continuum damage modifications to the model allowed for all aspects of creep curves to be captured by the model. A slightly different strain partitioning strategy was used by Wang et al. [20] as total strain was decomposed in to elastic and inelastic components, with the latter further decomposed into viscoplastic and creep strains. An ONERA type flow rule [7] was used for the description of viscoplastic strains.

A commercial Nickel based superalloy is considered in the present work, namely RR1000 (used widely in Rolls-Royce aeroengine components). Due to the commercially sensitive nature of the material data all axes have been normalised and material parameter values are not presented. Readers interested in a more quantifiable treatment of combined viscoelasticity and viscoplasticity are encouraged to read the author's previous work [18], wherein a similar model is applied in order to study a wide range of time dependencies in a chromium steel (P91). It is, of course, important to note that the martensitic microstructure of P91 differs significantly from the $\gamma / \gamma /$ structure of RR1000. At the macro scale however similar general behaviours are observed, particularly in relation to time dependencies over a wide range of loading rates. The phenomenological (but thermodynamically consistent) approaches developed in Benaarbia et al. [18] and in the present work are therefore applicable to both materials. Note also that only uniaxial loadings are 
considered in the present work, meaning that differences in the material behaviours in terms of anisotropy (through, for example, distortional hardening) are not represented.

\section{Model Formulation and Thermodynamic Basis}

The model developed in the present work uses the formalism of the thermodynamics of irreversible processes, which is excellently summarised in the work of Chaboche and Lemaitre [21]. Helmholtz free energy $(\psi)$ is expressed by equation (1) by assuming a decomposition of elastic $\left(\psi_{e}\right)$, viscoelastic $\left(\psi_{V E}\right)$, and viscoplastic $\left(\psi_{V P}\right)$ components, or $\psi=\psi_{e}+\psi_{V E}+\psi_{V P}$. This mirrors the decomposition of total strain $\boldsymbol{\epsilon}$ into elastic $\left(\boldsymbol{\epsilon}_{e}\right)$, viscoelastic $\left(\boldsymbol{\epsilon}_{V E}\right)$, and viscoplastic $\left(\boldsymbol{\epsilon}_{V P}\right)$ components, such that $\boldsymbol{\epsilon}=\boldsymbol{\epsilon}_{e}+\boldsymbol{\epsilon}_{V E}+\boldsymbol{\epsilon}_{V P}$. Note that, in the remainder of the present work, bold faced symbols will be used to denote tensor quantities and all other symbols will refer to scalar quantities.

$$
\begin{aligned}
\psi= & \frac{1}{2}\left(\boldsymbol{\epsilon}-\boldsymbol{\epsilon}_{V E}-\boldsymbol{\epsilon}_{V P}\right): \boldsymbol{C}_{e}:\left(\boldsymbol{\epsilon}-\boldsymbol{\epsilon}_{V E}-\boldsymbol{\epsilon}_{V P}\right)+\frac{1}{3} \sum_{i=1}^{n_{V E}} C_{i}^{V E} \boldsymbol{\alpha}_{i}^{V E}: \boldsymbol{\alpha}_{i}^{V E}+\ldots \\
& \frac{Q^{V E}}{b^{V E}}\left(b^{V E_{r} V E}+\exp \left(-b^{V E_{r} V E}\right)\right)+\frac{1}{3} \sum_{i=1}^{n_{V P}} C_{i}^{V P} \boldsymbol{\alpha}_{i}^{V P}: \boldsymbol{\alpha}_{i}^{V P}+\frac{Q^{V P}}{b^{V P}}\left(b^{V P} r_{r}^{V P}+\exp \left(-b^{V P} r_{r}^{V P}\right)\right)
\end{aligned}
$$

In equation (1), $C_{e}$ is a fourth order stiffness tensor which describes the (linear) relationship between the elastic strain component and the Cauchy stress tensor $\sigma$. Isotropic and kinematic terms are expressed in both the viscoelastic and viscoplastic components of $\psi$. This allows for limit/yield surfaces related to two deformation modes to be modified at different rates, resulting in a more general formulation. While a distinction is made between " $V E$ " and " $V P$ " terms, relating to viscoelastic and viscoplastic quantities respectively, the form of the free energy components is identical. In the interest of brevity in descriptions, $\beta$ is used as a placeholder term which which may be replaced by either $V E$ and $V P$ to refer to either viscoelastic or viscoplastic expressions, respectively. As in the work of Chaboche, kinematic hardening (back stress, $\chi^{\beta}$ ) is expressed by the summation of multiple $\left(n_{\beta}\right)$ components. $C_{i}^{\beta}$ is a hardening modulus term relating to the $i^{\text {th }}$ component of back stress. This acts on a kinematic related strain like quantity $\boldsymbol{\alpha}_{i}^{\beta}$. Isotropic hardening is modelled through the evolution of a scalar (drag) stress $R^{\beta}$. An exponential function is assumed for the description of $R^{\beta}$ (which varies with the strain like scalar quantity $r^{\beta}$ ). In these exponential expressions, $Q^{\beta}$ is a saturation value and $b^{\beta}$ controls how quickly this value is achieved.

State laws for thermodynamic forces can be determined from equation (1) by taking partial derivatives with respect to corresponding state variables. Cauchy stress $(\sigma)$ is the thermodynamic force relating to $\epsilon_{e}$, leading to equation (2).

$$
\sigma=\frac{\partial \psi}{\partial \epsilon_{e}}=C_{e}:\left(\epsilon-\epsilon_{V E}-\epsilon_{V P}\right)
$$

Thermodynamic forces relating to other strain components are here denoted by $\boldsymbol{X}_{\beta}$, thereby allowing equation (3) to be developed.

$$
\begin{aligned}
& \boldsymbol{X}_{V E}=\frac{\partial \psi}{\partial \epsilon_{V E}}=-\sigma \\
& \boldsymbol{X}_{V P}=\frac{\partial \psi}{\partial \epsilon_{V P}}=-\sigma
\end{aligned}
$$

Back stresses, $\chi_{i}^{\beta}$, are the thermodynamic forces related to the strain like quantities $\alpha_{i}^{\beta}$ (see equation (4)). Note that the expressions in equation (4) are identical to the well known Prager type hardening law, which has been widely used in the past to provide a simple description of kinematic effects. 


$$
\begin{aligned}
& \chi_{i}^{V E}=\frac{\partial \psi}{\partial \boldsymbol{\alpha}_{i}^{V E}}=\frac{2}{3} C_{i}^{V E} \boldsymbol{\alpha}_{i}^{V E} \\
& \chi_{i}^{V P}=\frac{\partial \psi}{\partial \boldsymbol{\alpha}_{i}^{V P}}=\frac{2}{3} C_{i}^{V P} \boldsymbol{\alpha}_{i}^{V P}
\end{aligned}
$$

Finally, state functions for the drag stress terms $R^{\beta}$ are given in equation (5).

$$
\begin{aligned}
& R^{V E}=\frac{\partial \psi}{\partial r^{V E}}=Q^{V E}\left(1-\exp \left(-b^{V E_{r} V E}\right)\right) \\
& R^{V P}=\frac{\partial \psi}{\partial r^{V P}}=Q^{V P}\left(1-\exp \left(-b^{V P} r^{V P}\right)\right)
\end{aligned}
$$

This completes state function determination for the viscoelastic - viscoplastic model. In order to define evolution functions a dissipation potential, $\phi$, must be determined. It is common to formulate $\phi$ in terms of state variables (here strain or strain like quantities, such as $\alpha^{\beta}$ ), however it is more convenient here to transform $\phi$ to its dual, denoted as $\phi^{*}$. This can be achieved using the Legendre-Fenchel (or convex conjugate) transformation. Note that $\phi^{*}$ is defined in terms of thermodynamic forces, such as the stress quantities described above. Before specifying $\phi^{*}$ several supporting terms must be outlined. The model developed here implements two limiting (or "yield") surfaces to describe viscoelastic and viscoplastic phenomenon, denoted as $f^{V E}$ and $f^{V P}$ respectively (see equations (6) and (7)). Both surfaces make use of the well known second invariant operator $\left(J_{2}(\right.$.$) , see equation (8)) to evaluate a "distance" measure in stress$ space. Distances are determined between the centre of a limiting surface (defined by $\chi^{\beta}$ ) and the deviatoric component of the stress tensor $\left(\sigma^{\prime}\right)$. Distance measures are modified by related isotropic hardening terms $R^{\beta}$ and scalar parameters relating to the initial size of the limiting surfaces $\left(R_{0}^{V E}\right.$ and $\sigma_{y}$ for viscoelastic and viscoplastic surfaces, respectively). In defining $R_{0}^{V E}$ an equilibrium stress is specified, meaning that stress cannot relax below this value and loadings below this level will be entirely elastic in nature. The physical significance of this parameter is that obstacles may exist in materials which will support some non-zero stress (by preventing dislocation motion, for example), even at elevated temperature. Note also that in rate independent plasticity the consistency condition would apply, meaning that $f^{\beta} \leq 0$ always applies $\left(f^{\beta}=0\right.$ only during plasticity). In time dependent formulations the consistency condition can be violated as an "overstress" can be defined by the model.

$$
\begin{gathered}
f^{V E}=J_{2}\left(\sigma^{\prime}-\chi^{V E}\right)-R^{V E}-R_{o}^{V E} \\
f^{V P}=J_{2}\left(\sigma^{\prime}-\chi^{V P}\right)-R^{V P}-\sigma_{y} \\
J_{2}(\boldsymbol{X})=\left(\frac{3}{2} \boldsymbol{X}: \boldsymbol{X}\right)^{1 / 2}
\end{gathered}
$$

Two flow potentials $\left(F^{\beta}\right.$, see equation (9)) are defined for the limiting surfaces which include dynamic recovery components of the $\chi^{\beta}$ quantities. The rate of dynamic recovery is specified by the parameter $\gamma_{i}^{\beta}$. The dual dissipation potential may now be defined, as shown in equation (10). Hyperbolic sine type flow rules are used to describe time dependencies in both limiting surfaces and require the definition of three material parameters each $\left(A^{\beta}, K^{\beta}\right.$, and $\left.m^{\beta}\right)$. 


$$
\begin{gathered}
F^{V E}=f^{V E}+\frac{1}{2} \sum_{i=1}^{n_{V E}} \frac{\gamma_{i}^{V E}}{C_{i}^{V E}} J_{2}^{2}\left(x_{i}^{V E}\right) \\
F^{V P}=f^{V P}+\frac{1}{2} \sum_{i=1}^{n_{V P}} \frac{\gamma_{i}^{V P}}{C_{i}^{V P}} J_{2}^{2}\left(x_{i}^{V P}\right) \\
\phi^{*}=\int A^{V E}\left[\sinh \left(\frac{f^{V E}}{K^{V E}}\right)\right]^{m^{V E}} d F_{V E}+\int A^{V P}\left[\sinh \left(\frac{f^{V P}}{K^{V P}}\right)\right]^{m^{V P}} d F_{V P}
\end{gathered}
$$

Evolution equations for the state variables can now found be taking partial derivatives of equation (10) with respect to corresponding thermodynamic forces. In the case of isotropic quantities, it can be shown that the strain rate term $r^{\beta}$ is equivalent to the monotonically increasing quantity $\lambda_{\beta}$ (see equation (11)), where $\dot{\lambda}_{\beta}$ is the absolute value of the corresponding strain rate term. In this sense, $\lambda_{\beta}$ represents accumulated (monotonic) viscoelastic and viscoplastic strain.

$$
\begin{aligned}
& r^{V E}=\frac{\partial \phi^{*}}{\partial R_{V E}}=\dot{\lambda}_{V E} \\
& r^{V P}=\frac{\partial \phi^{*}}{\partial R_{V P}}=\dot{\lambda}_{V P}
\end{aligned}
$$

A similar process is used to find viscous strain rates $\left(\dot{\boldsymbol{\epsilon}}_{\beta}\right)$, however the chain rule is implemented in completing the differentiation (see equation (12)). Due to the presence of $f^{\beta}$ in $F_{\beta}$ it is usually the case that the derivative of $F_{\beta}$ with respect to a thermodynamic force will result in a unit normal vector, $N_{\beta}$. It is now apparent from equation (12) that $\lambda_{\beta}$ is a plastic multiplier term. The process is repeated for kinematic hardening components (see equation (12) for a detailed application to $\dot{\alpha}_{i}^{V E}$ ), however the dynamic recovery components in $F_{\beta}$ now give rise to an additional term when $F_{\beta}$ is differentiated with respect to $\chi_{\beta}$. By recalling state equations (see equation (4)) functions for $\dot{\boldsymbol{\alpha}}_{i}^{\beta}$ may be used to find the more useful expressions given in equations (14) and (15) (for viscoelastic and viscoplastic terms, respectively).

$$
\begin{aligned}
\dot{\boldsymbol{\epsilon}}_{V E}= & \frac{\partial \phi^{*}}{\partial \boldsymbol{X}_{V E}}=-\frac{\partial \phi^{*}}{\partial F_{V E}} \frac{\partial F_{V E}}{\partial \boldsymbol{X}_{V E}}=A^{V E}\left[\sinh \left(\frac{f^{V E}}{K^{V E}}\right)\right]^{m^{V E}} \boldsymbol{N}_{V E}=\dot{\lambda}_{V E} N_{V E} \\
\dot{\boldsymbol{\epsilon}}_{V P}= & \frac{\partial \phi^{*}}{\partial \boldsymbol{X}_{V P}}=-\frac{\partial \phi^{*}}{\partial F_{V P}} \frac{\partial F_{V P}}{\partial \boldsymbol{X}_{V P}}=A^{V P}\left[\sinh \left(\frac{f^{V P}}{K^{V P}}\right)\right]^{m^{V P}} \boldsymbol{N}_{V P}=\dot{\lambda}_{V P} \boldsymbol{N}_{V P} \\
\dot{\boldsymbol{\alpha}}_{i}^{V E}= & \frac{\partial \phi^{*}}{\partial \chi_{V E}}=-\frac{\partial \phi^{*}}{\partial F_{V E}} \frac{\partial F_{V E}}{\partial \chi_{V E}}=-\dot{\lambda}_{V E}\left(-\frac{3}{2} \frac{\left(\sigma^{\prime}-\chi_{V E}\right)}{J_{2}\left(\sigma^{\prime}-\chi_{V E}\right)}+\frac{3}{2} \frac{\gamma_{i}^{V E}}{C_{i}^{V E}} \chi_{i}^{V E}\right) \\
= & {\left[\boldsymbol{N}_{V E}-\frac{3}{2} \frac{\gamma_{i}^{V E}}{C_{i}^{V E}} \chi_{i}^{V E}\right] \dot{\lambda}_{V E} } \\
= & \dot{\boldsymbol{\epsilon}}_{V E}-\frac{3}{2} \frac{\gamma_{i}^{V E}}{C_{i}^{V E}} \chi_{i}^{V E} \dot{\lambda}_{V E} \\
& \dot{\chi}_{i}^{V E}=\frac{2}{3} C_{i}^{V E} \dot{\boldsymbol{\alpha}}_{i}^{V E}=\frac{2}{3} C_{i}^{V E} \dot{\boldsymbol{\epsilon}}_{V E}-\gamma_{i}^{V E} \chi_{i}^{V E} \dot{\lambda}_{V E}
\end{aligned}
$$




$$
\dot{\chi}_{i}^{V P}=\frac{2}{3} C_{i}^{V P} \dot{\boldsymbol{\alpha}}_{i}^{V P}=\frac{2}{3} C_{i}^{V P} \dot{\boldsymbol{\epsilon}}_{V P}-\gamma_{i}^{V P} \chi_{i}^{V P} \dot{\lambda}_{V P}
$$

A viscoelastic viscoplastic model has been formulated thermodynamically. Several simplifying conditions may now be applied to limit the number of required material parameters and to reduce the complexity of parameter determination procedures. Furthermore the uniaxial form of the model, that will be used in the remainder of the present, work can be derived. To ensure that limit surfaces always remain concentric a single back stress, evolving with the accumulation of viscoplastic strain, can be assumed (that is to say, $\chi_{V E}=\chi_{V P}$ ). This also ensures that viscoelastic and viscoplastic strains will accumulate in the same direction, as with this condition it is always true that $N_{V E}=N_{V P}$. Descriptions of $\chi$ are also limited to two Armstrong-Frederick components, as this is usually sufficient for most materials. Isotropic evolution of the viscoelastic limiting surface is also neglected as this is difficult to determine with any confidence from the limited experimental data used in the present work. By applying these assumptions and reformulating tensor quantities a uniaxial form of the model may be derived, as shown in equation (16). It is important to note here that the function $\operatorname{sgn}()$ returns a value of 1 if the argument is positive, a value of -1 is the argument is negative, and a value of 0 if the argument is 0 . McCauley brackets $(\langle\rangle$.$) will only return a$ non-zero value if the argument is positive. In order to aid comprehension, a schematic of the model can be found in figure 1, showing the location of the two limiting surfaces in the deviatoric stress (or $\pi$ ) plane. Note that only uniaxial loading is considered in the present work (in the direction of $\sigma_{1}$ ), meaning that $\chi$ (which is here equivalent to $\chi_{V P}$ and $\chi_{V E}$ ) accumulates in this direction only.

$$
\begin{aligned}
\text { Strain Decomposition:- } & \epsilon=\epsilon_{e}+\epsilon_{V E}+\epsilon_{V P} \\
\text { Stress:- } & \sigma=E\left(\epsilon-\epsilon_{V E}-\epsilon_{V P}\right) \\
\text { Viscoelastic Limit Function:- } & f^{V E}=J_{2}(\sigma-\chi)-R_{o}^{V E} \\
\text { Viscoplastic Yield Function:- } & f^{V P}=J_{2}(\sigma-\chi)-R-\sigma_{y} \\
\text { Isotropic Hardening (Drag Stress):- } & R=b(Q-R) \dot{\lambda}_{V P} \\
\text { Kinematic Hardening (Back Stress):- } & \chi_{i}=C_{i} \dot{\epsilon}_{V P}-\gamma_{i} \chi_{i} \dot{\lambda}_{V P} \\
\text { Back Stress Decomposition:- } & \chi=\sum_{i=1}^{2} \chi_{i} \\
\text { Viscoelastic Strain Rate:- } & \dot{\epsilon}_{V E}=\left\langle A^{V E}\left[\sinh \left(\frac{f^{V E}}{K}\right)\right]^{m^{V E}}\right\rangle \operatorname{sgn}(\sigma-\chi) \\
\text { Accumulated Viscoelastic Strain:- } & \dot{\lambda}_{V E}=\left|\dot{\epsilon}_{V E}\right| \\
\text { Viscoplastic Strain Rate:- } & \dot{\epsilon}_{V P}=\left\langle A^{V P}\left[\sinh \left(\frac{f^{V P}}{K}\right)\right]^{m^{V P}}\right\rangle \operatorname{sgn}(\sigma-\chi) \\
\text { Accumulated Viscoplastic Strain:- } & \dot{\lambda}_{V P}=\left|\dot{\epsilon}_{V P}\right|
\end{aligned}
$$




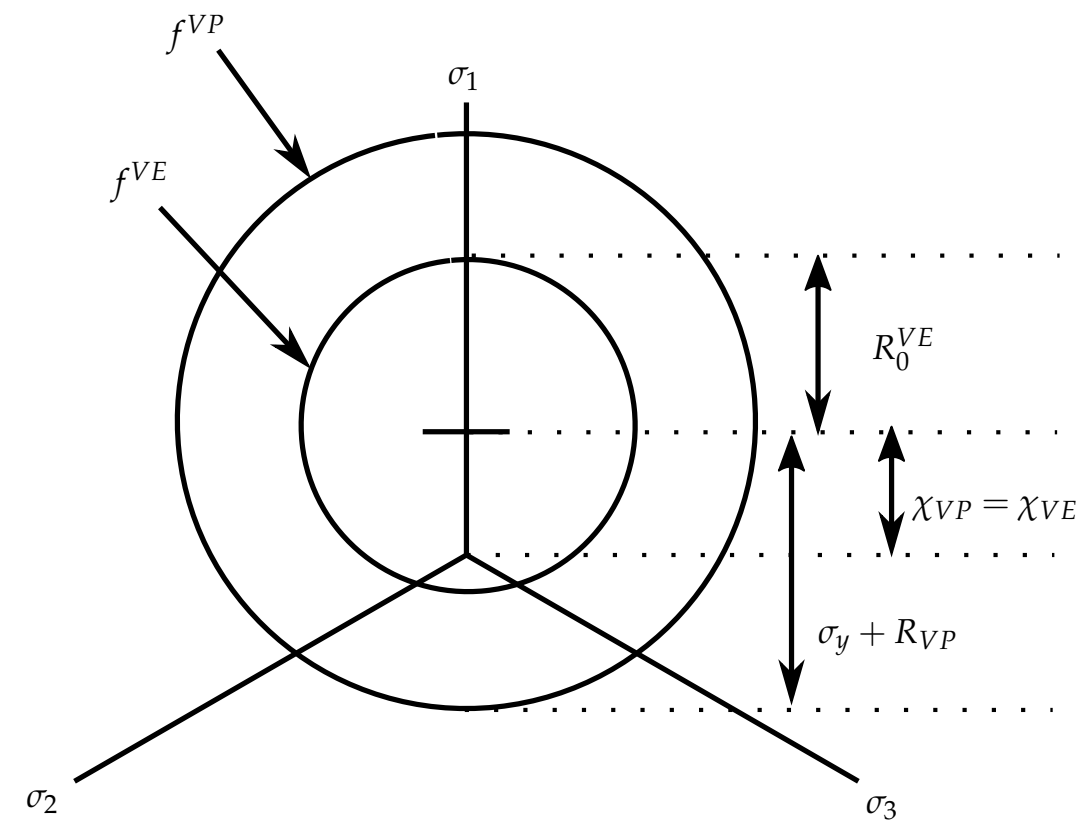

Figure 1: A schematic representation of the viscoelastic - viscoplastic model with simplifying assumptions applied, showing relative positions of viscoelastic and viscoplastic limiting surfaces (assuming uniaxial loading in $\sigma_{1}$ ).

\section{Material Parameter Determination}

The application of the simplifying assumptions described towards the end of section 2 means that 15 material parameters are required to apply the viscoelastic - viscoplastic model. For clarity, these are $E$ (Young's modulus), $\sigma_{y}$ (the initial size of the viscoplastic yield surface), $R_{o}^{V E}$ (the initial size of the viscoelastic limit surface), $b$ and $Q$ (which describe isotropic hardening in the viscoplastic surface), $C_{1}, \gamma_{1}, C_{2}$, and $\gamma_{2}$ (which describe kinematic hardening of both limiting surfaces), $A^{V P}, K^{V P}$, and $m^{V P}$ (which describe time dependencies in the viscoplastic surface), and $A^{V E}, K^{V E}$, and $m^{V E}$ (which describe time dependencies in the viscoelastic surface). The material model is highly non-linear with many degrees of freedom in the 15 material parameters, suggesting that careful thought is required in order to determine good initial estimates of the material parameters. These estimates can be fine tuned in subsequent optimisation procedures.

Estimating material parameters relating to isotropic and kinematic hardening terms can be accomplished using the Cottrell stress partitioning method [22]. This method is well known so only a brief review is given here, however a more detailed treatment can be found in the author's previous work [18]. A schematic representation of the method can be found in figure 2. Closed hysteresis loops from cyclic tests are analysed and, through linear regression, the linear elastic region is determined ( $B-C$ in figure 2$)$. It is worth noting that, while in viscoelastic formulations this region will not strictly be linear, the assumption of linearity is broadly applicable in regions of monotonic loading for the purposes of material parameter estimation. The limits of the elastic region ( $\sigma_{e l}^{+}$in tension and $\sigma_{e l}^{-}$in compression) define the size and position of the yield locus on the $\pi$ plane. Assuming a von Mises material with no distortional hardening (or similar), the centre of the yield surface can be found and hence $\chi$ and $R$ estimated. By noting the plastic strain $\left(\epsilon_{p}\right)$ from the width of the hysteresis loop relationships between $R \& \epsilon_{p}$ and $\chi \& \epsilon_{p}$ may be found. These in turn may be exploited using the functions in equation (16) to estimate hardening law parameters. Figure 2 also defines a viscous (time dependent) "overstress" $\sigma_{v}$, identifiable as some non-linearity in the hysteresis loop at load reversal. It is vital to note that, in the viscoelastic - viscoplastic model developed here, overstresses are 
developed on both limiting surfaces.

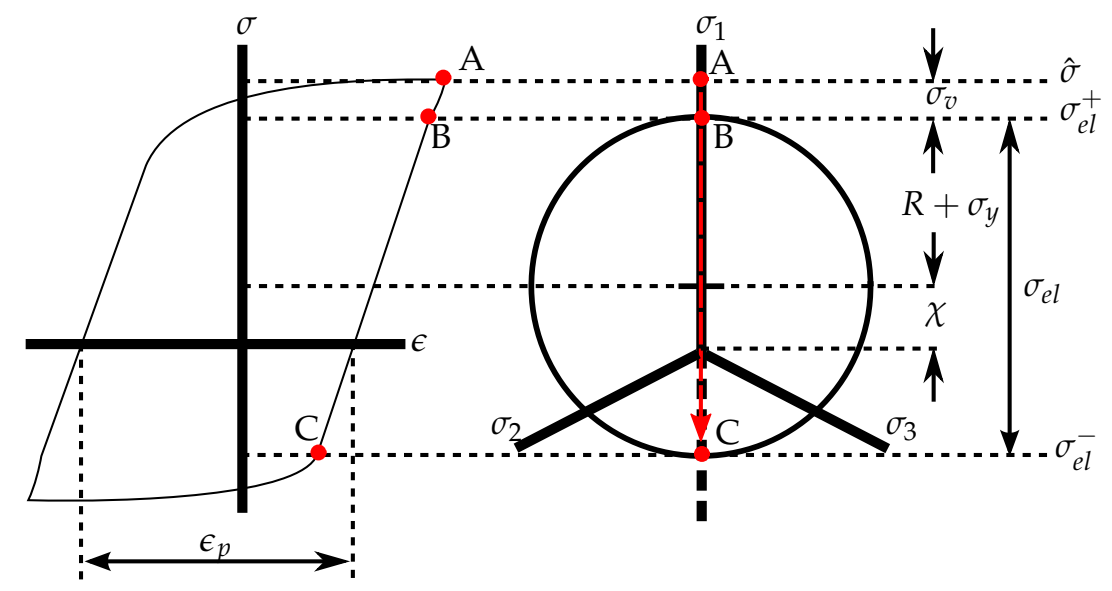

Figure 2: An overview of the Cottrell stress partitioning method [22], assuming a viscoplastic strain decomposition.

While the derivation of $b$ and $Q$ from $R / \epsilon_{p}$ data (which is extracted by the Cottrell method) is fairly straightforward, more care is required for kinematic terms. If only monotonic loadings are considered it is easy to show that $\dot{\lambda}_{V P}=\dot{\epsilon}_{V P}$. If this is the case, the back stress expressions in equation (16) can be integrated to give equation (17). Examination of equation (17) indicates that the function will saturate. This saturation value is here denoted as $\bar{\chi}_{i}$ and is defined by equation (18). Values of $\bar{\chi}_{i}$ for each component of back stress (recall two Armstrong-Frederick components are used in the present work) can be estimated using the values found by the Cottrell method. Considering an intermediate value of back stress $\left(\tilde{\chi}_{i}\right.$, a value of back stress prior to saturation) taken from the monotonic loading region at a strain $\tilde{\epsilon}_{p}$, equation (19) may be developed which expresses $\tilde{\chi}_{i}$ in terms of $\bar{\chi}_{i}$. This may be rearranged to give equation (20) which, along with equation (18), gives sufficient conditions to estimate $C_{i}$ and $\gamma_{i}$.

$$
\begin{gathered}
\chi_{i}=\frac{1}{\gamma_{i}} C_{i}\left[1-\exp \left(-\gamma_{i} \epsilon_{p}\right)\right] \\
\bar{\chi}_{i}=\frac{C_{i}}{\gamma_{i}} \\
\tilde{\chi}_{i}=\frac{C_{i}}{\gamma_{i}}\left[1-\exp \left(-\gamma_{i} \tilde{\epsilon}_{p}\right)\right]=\bar{\chi}_{i}\left[1-\exp \left(-\gamma_{i} \tilde{\epsilon}_{p}\right)\right] \\
\gamma_{i}=-\frac{1}{\tilde{\epsilon}_{p}} \ln \left(1-\frac{\tilde{\chi}_{i}}{\bar{\chi}_{i}}\right)
\end{gathered}
$$

The estimation of viscous material parameters is, generally speaking, more difficult than hardening parameters. This is particularly true in the case of the sinh flow rules applied in the present work's model. Relevant parameters are here estimated by interpreting stress relaxation data during hold periods in cyclic tests. For viscoelastic related parameters, hold strains should be chosen such that peak stresses are less than the "conventional" yield stress (i.e. what would be normally reported if an elastic-viscoplastic model formulation were to be assumed) of the material. Under the assumed viscoelastic - viscoplastic formulation, this would imply that $\epsilon_{V P}=0$, meaning that the evolution $\epsilon_{V E}$ can be estimated from the hold data by equation (21), where $\epsilon$ is the hold (or total) strain and $\sigma$ is an instantaneous measure of stress during the relaxation period. Clearly $\epsilon_{V E}$ will vary with time (the relaxation of $\sigma$ is achieved through the accumulation of $\epsilon_{V E}$ here) and a smoothing function (power laws of the form $y=A x^{b}$ are often sufficient) can be fitted such that $\dot{\epsilon}_{V E}$ can be estimated. Returning attention to the expression of the flow rule in equation (16), 
an evaluation of $f^{V E}$ is now required. For the reasons outlined in the description of figure $2, f^{V E}$ can be interpreted as a viscoelastic overstress (i.e. the difference between the instantaneous measure of stress and the current size of the limit surface). The size of the viscoelastic surface, $R_{0}^{V E}$, can be approximated by observing the equilibrium stress to which stresses relax over long hold periods. The overstress, $\sigma_{V E}$, can therefore be estimated by taking the difference between the instantaneous value of $\sigma$ and $R_{0}^{V E}$. In order to complete the process and find initial estimates of $A^{V E}, K^{V E}$, and $m^{V E}$ several more assumptions must be made. The form of the flow rule in equation (16) suggests that $K^{V E}$ acts to normalise $\sigma_{V E}$, therefore it is reasonable to assume that $K^{V E}$ is of the same order of magnitude as $\sigma_{V E}$ (in practice an average value of $\sigma_{V E}$ may be assumed). The identity $\sinh (x)=(\exp (x)-\exp (-x)) / 2$ may then be considered and, assuming that $\exp \left(\sigma_{V E} / K^{V E}\right)>>1 / \exp \left(\sigma_{V E} / K^{V E}\right)$, equation (22) developed. By assuming a value for $K^{V E}$ and plotting $\left(\sigma_{V E} / K^{V E}\right)$ versus the natural logarithm of $\dot{\epsilon}_{V E}$, estimates of $A^{V E}$ and $m^{V E}$ may be found from the intercept and gradient, respectively. Note that the choice of $K^{V E}$ may be validated by observing how it linearises the plot of $\left(\sigma_{V E} / K^{V E}\right)$ versus the natural logarithm of $\dot{\epsilon}_{V E}$. A similar process may be used to estimate $A^{V P}, K^{V P}$, and $m^{V P}$, however stress relaxation data should be collected for higher hold strains and viscoplastic overstress should be approximated as the difference between instantaneous stress and $\sigma_{y}$ (the initial size of the viscoplastic yield locus) plus the saturated value of the back stresses (which can be determined by taking the ratio of $C_{i}$ to $\gamma_{i}$ ). It is important to note that strain rates should also be corrected to account for viscoelastic effects in the viscoplastic domain $\left(\epsilon_{V P}=\epsilon-\epsilon_{e} \epsilon_{V E}\right)$, however this is not difficult once estimates for $A^{V E}, K^{V E}$, and $m^{V E}$ have been made.

$$
\begin{gathered}
\epsilon_{V E}=\epsilon-\epsilon_{e}=\epsilon-\frac{\sigma}{E} \\
\ln \left(\dot{\epsilon}_{V E}\right)=\ln \left(A^{V E}\right)+m^{V E}\left(\frac{\sigma_{V E}}{K^{V E}}\right)-m^{V E} \ln (2)
\end{gathered}
$$

\section{Results}

\subsection{Experimental Results and Initial Observations}

In the present work parameters for the viscoelastic - viscoplastic model are determined for RR1000 at $750^{\circ} \mathrm{C}$ using a very limited experimental data set. All tests use standard loading waveforms which a routinely applied in many industries for basic material characterisation exercises. Although some creep data (minimum creep strain rates) is used for verification of viscous parameters most of the parameter determination processes can be completed using strain controlled cyclic data with hold periods at peak loads. A summary of this data is given in figure 3.

Two test types are considered in the present work, namely a fully reversed strain controlled cyclic test (resulting in 19 loading cycles) in which hold periods where enforced at peak strains of $0.8 \%$ and $-0.8 \%$ (i.e. a trapezoidal waveform is applied) and a stress relaxation test, wherein a specimen was loaded to $0.4 \%$ strain (stresses at this strain are still below the apparent yield of the material). Note that a similar test with a hold strain of $0.3 \%$ is used later for additional verification of the model in the viscoelastic regime. Selected cyclic data at $750^{\circ} \mathrm{C}$ is presented in figure 3. It can be seen that back stresses saturate quickly (within the first three loading cycles) and a stabilised hysteresis loop is developed with little to no secondary isotropic hardening. A comparison of monotonic and first cycle tensile strain hold responses is presented in figure 3 (b), (c), and (d), showing data from both cyclic plasticity and stress relaxation tests. Figure 3 (b) clearly shows that the stress relaxation test is at a load which would normally be considered to be in the elastic domain of the material (note that $\sigma / \epsilon$ behaviour is approximately linear prior to stress relaxation), suggesting that this behaviour would be impossible to capture in elastic - viscoplastic model formulations without adopting artificially low yield stress values. Time dependencies are explored in figure 3 (c) and (d), with the period considered in the latter approximately one hundredth of that considered in the former (hold periods used in cyclic tests are much shorter than those used in stress relaxation tests). It can be seen that, while little viscoelastic stress relaxation is observed over the relatively short hold period used in the 
cyclic tests (figure $3(\mathrm{~d})$ ), a significant amount of viscoelastic stress relaxation is observed over the full stress relaxation test (figure 3 (c)). Cyclic data for RR1000 at $400^{\circ} \mathrm{C}$ is also presented here (see figure 4) as these results will be used in section 5 to explore an-isothermal extensions to the model. Strain limits applied at $400^{\circ} \mathrm{C}$ are the same as those used at $750^{\circ} \mathrm{C}$ and no stress relaxation is observed.

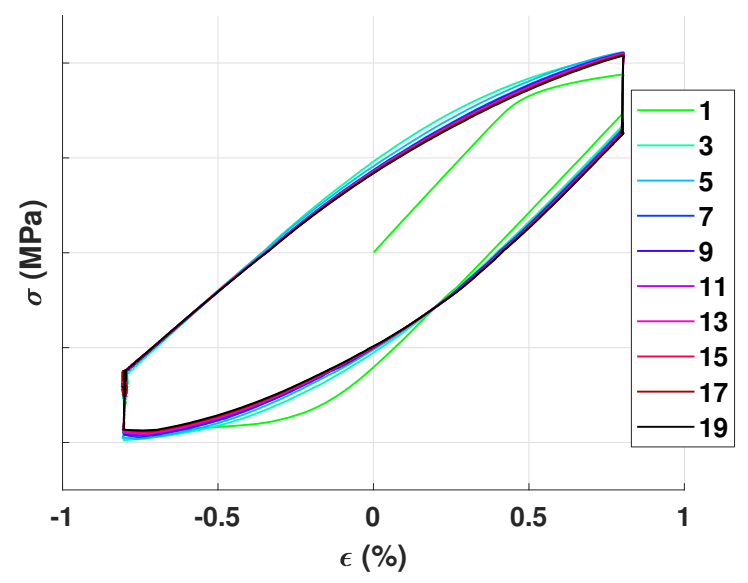

(a)

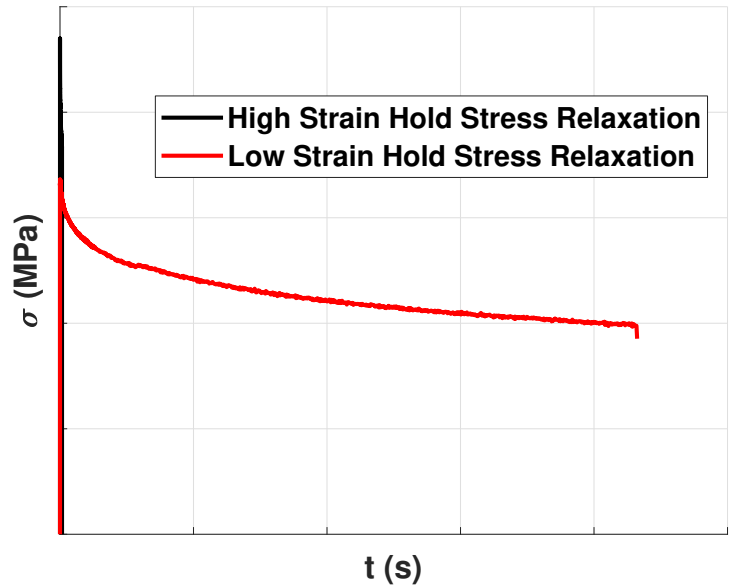

(c)

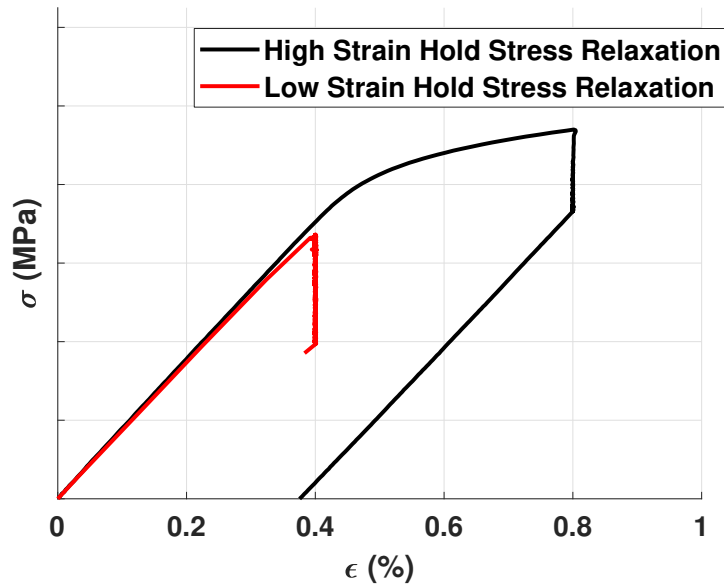

(b)

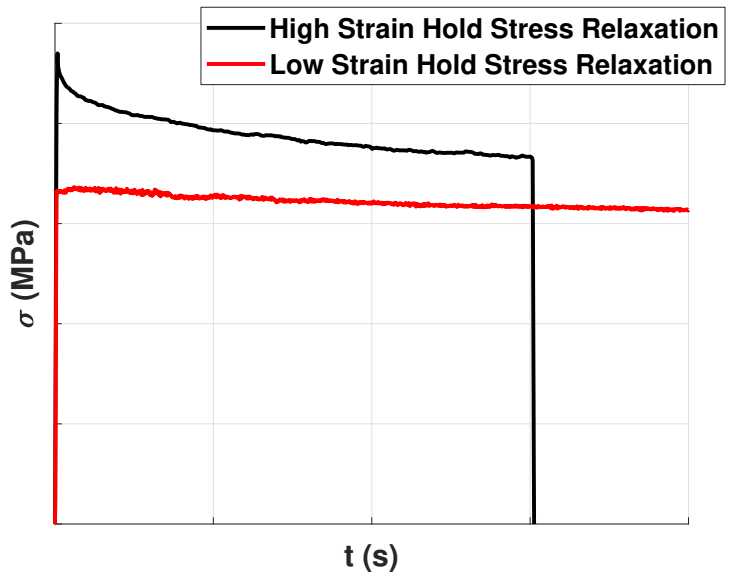

(d)

Figure 3: A summary of $750^{\circ} \mathrm{C}$ experimental data used in the present work, showing (a) general cyclic behaviour over 20 fully reversed cycles with hold periods at tension and compression, (b) a comparison of $\sigma / \epsilon$ behaviour for high and low hold strain stress relaxation loadings, (C) a comparison of $\sigma / t$ behaviour for high and low hold strain stress relaxation loadings, and (d) a comparison of $\sigma / t$ behaviour for high and low hold strain stress relaxation loadings over a smaller time period. 


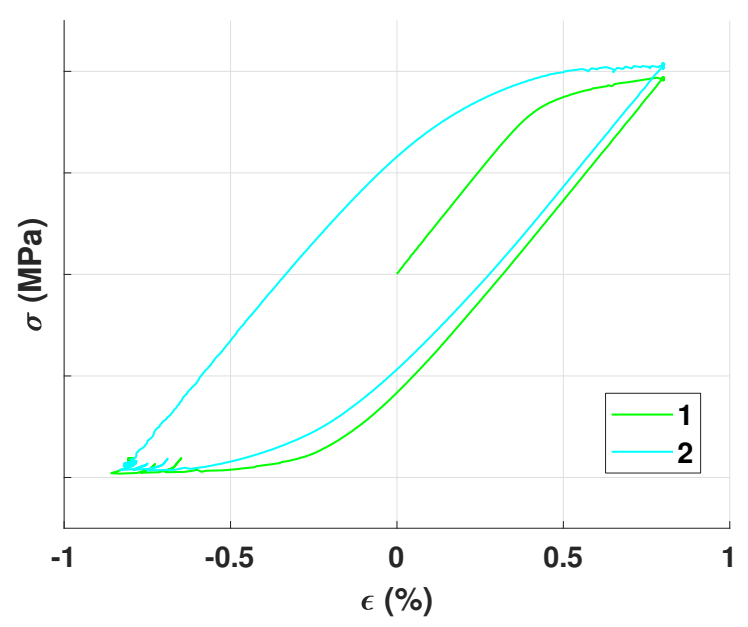

Figure 4: A summary of $400^{\circ} \mathrm{C}$ experimental data used in the present work, showing general cyclic behaviour over 2 fully reversed cycles with hold periods at tension and compression. Note the lack of any stress relaxation under hold conditions.

\subsection{Model Results}

The cyclic data presented in section 4.1 will now be used in conjunction with the methods discussed in section 3 to determine parameter values for RR1000 at $750^{\circ} \mathrm{C}$, assuming the viscoelastic - viscoplastic material model formulated in section 2. Attention will initially be focused on estimating hardening and viscous parameters from monotonic and relaxation data, respectively.

Figure 5 summarises the data used to estimate viscous material parameters $A^{V P}, K^{V P}, m^{V P}, A^{V E}, K^{V E}$ and $m^{V E}$. In figure 5 (a) and (c) viscoelastic and viscoplastic strains $\left(\epsilon_{V E}\right.$ and $\left.\epsilon_{V P}\right)$ are estimated from the low and high strain stress relaxation tests, respectively. Power law (of the form $y=A x^{B}$ ) functions are used to smooth this data and estimate strain rate terms. Overstresses ( $\sigma_{V E}$ and $\sigma_{V P}$ for viscoelastic and viscoplastic flow mechanisms, respectively) are determined by approximating the size of the corresponding limit surface during stress relaxation (see section 3). The size of the viscoplastic limit surface is taken to be $\sigma_{y}$ (which is determined by deviation from linearity in the monotonic data set) and the limit size of the viscoelastic surface, $R_{o}^{V E}$, is approximated as half of $\sigma_{y}$ (note this is below the minimum stress observed in stress relaxation tests). Overstress and strain rate parameters are used to construct figure $5(\mathrm{~b})$ and $(\mathrm{d})$ for viscoelastic and viscoplastic cases, respectively, leading to the estimation of related viscous material parameters. Note that, in figure 5 (b) and (d), strain rates predicted by the flow rules are compared to corresponding experimental data (interpreted using the methods described above). In the case of viscoelastic flows (figure 5 (b)) experimental creep data is also included and shows a good level of agreement with strain rates observed in the stress relaxation test and the strain rates predicted by the sinh flow rule. A deviation between the viscoelastic flow rule and the creep data (in the form of minimum creep strain rates observed in constant load tests) is observed for higher $\sigma_{V E}$ values in figure 5 (b), however it is noted that these rates are for overstresses that would require a stress above yield (as indicated by the dashed vertical line). 


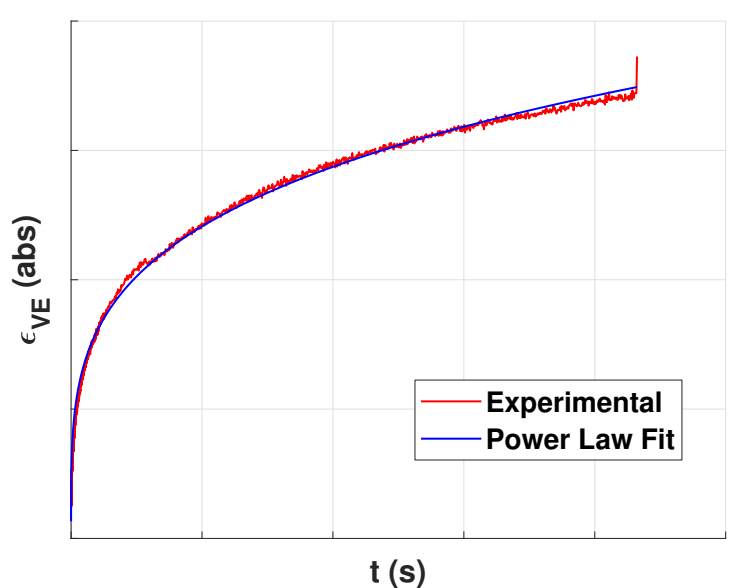

(a)

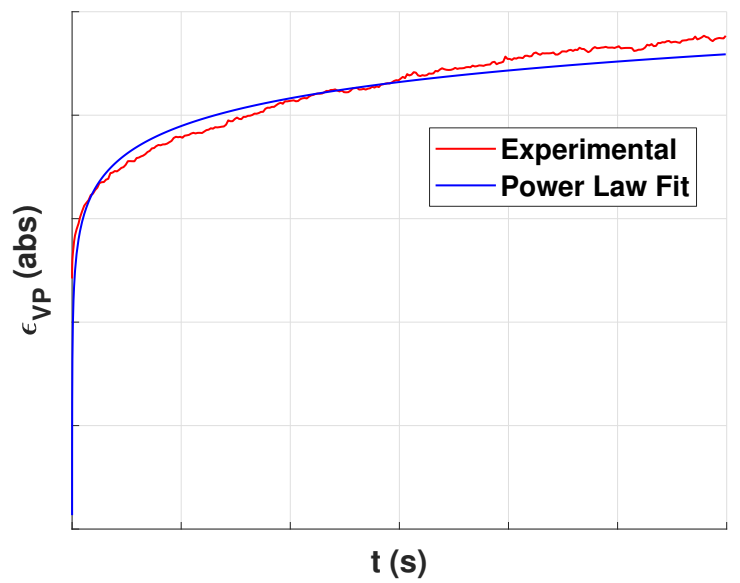

(c)

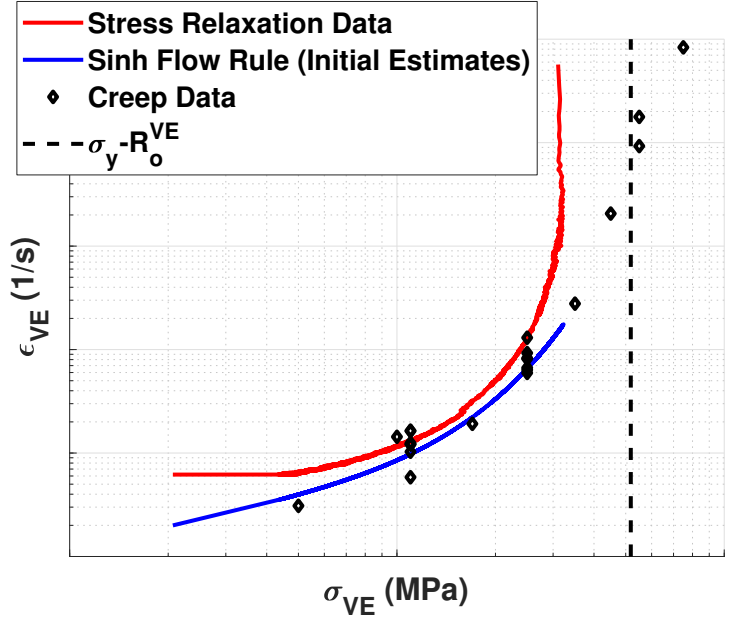

(b)

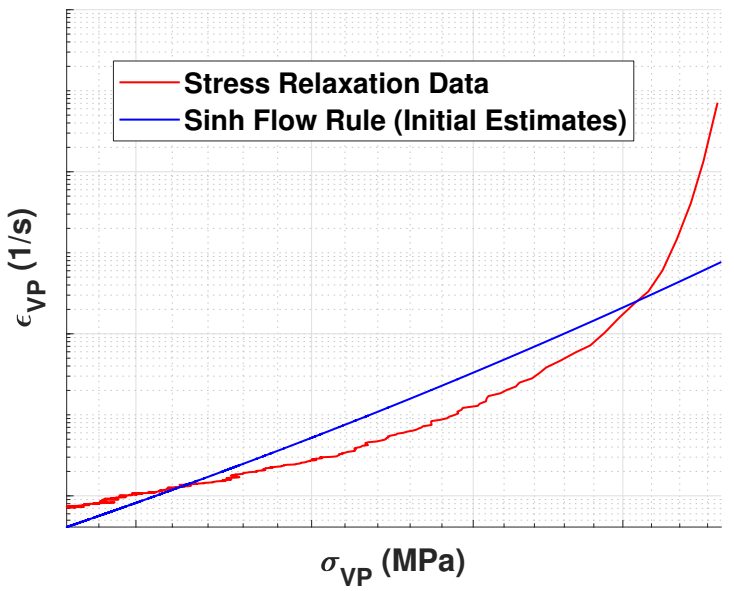

(d)

Figure 5: A summary of data interpretation methods used to determine initial estimates for viscoelastic and viscoplastic flow rule parameters. All data relatees to RR1000 at $750^{\circ} \mathrm{C}$. For viscoelastic parameters, (a) shows smoothed (power law fit) $\epsilon_{V E}$ data taken from stress relaxation tests. Derivatives of this function are used in (b) to find $\dot{\epsilon}_{V E}$, thereby allowing viscoelastic flow rule parameters to be estimated. A similar method is used for viscoplastic parameters, with smoothed relation data shown in (c) and flow rule fits in (d).

Armstrong-Frederick parameters $\left(C_{1}, \gamma_{1}, C_{2}\right.$, and $\left.\gamma_{2}\right)$ are determined using methods discussed above (assuming time independent behaviour), as shown in figure 6 (a). These initial estimates are generated using monotonic data from figure 3 (a) and, (along with the initial estimates of $A^{V P}, K^{V P}, m^{V P}, A^{V E}, K^{V E}$, and $m^{V E}$, are used to generate the blue curves in figure 6 (b) and (c) (showing $\sigma / \epsilon$ and $\sigma / t$ behaviours for monotonic and first stress relation loadings, respectively). Initial estimates of the material parameters have been optimised using Matlab's LSQNONLIN function (a Levenberg-Marquardt non-linear least squares algorithm) by defining a simple objective function that evaluates the difference between experimental and predicted stress magnitudes for the monotonic/first stress relaxation loading conditions. Figure 6 (b) and (c) also show the model predictions calculated using the optimised parameter set and a marked improvement is noted over the initial estimates. Finally, all 19 cycles of data are used in a final optimisation procedure (using the same algorithm and objective function as in the monotonic data) to refine all 15 material parameters. The fully calibrated model is used to predict material response for cycles 1,2,10, and 15 in figures 7 to 10, respectively. Excellent agreement between the model and experimental data is observed in both $\sigma / \epsilon$ and $\sigma / t$ behaviours. Partial verification of the model parameters is completed by comparing model predictions to creep data (figure 11) and two stress relaxation tests (figure 12). For creep data, deviations at high stresses 
(observed in figure 5 (b)) are reduced as viscoplastic strain rates are now represented (applicable when creep stresses are greater than $\sigma_{y}$ ). An excellent level of agreement is observed for stress relaxation data in figure 12 .

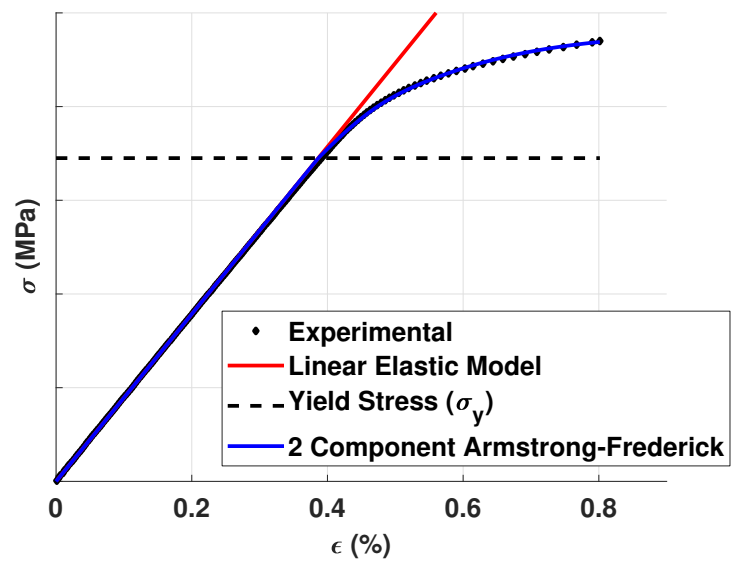

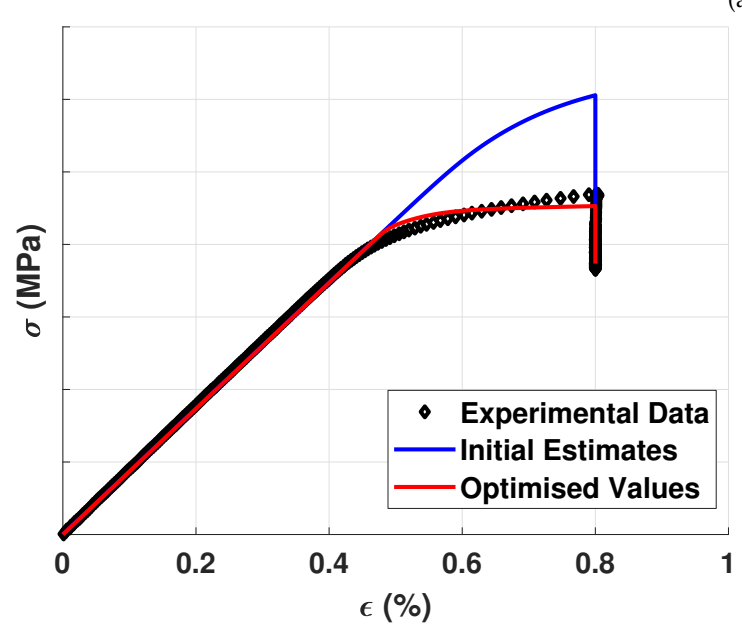

(b)

a)

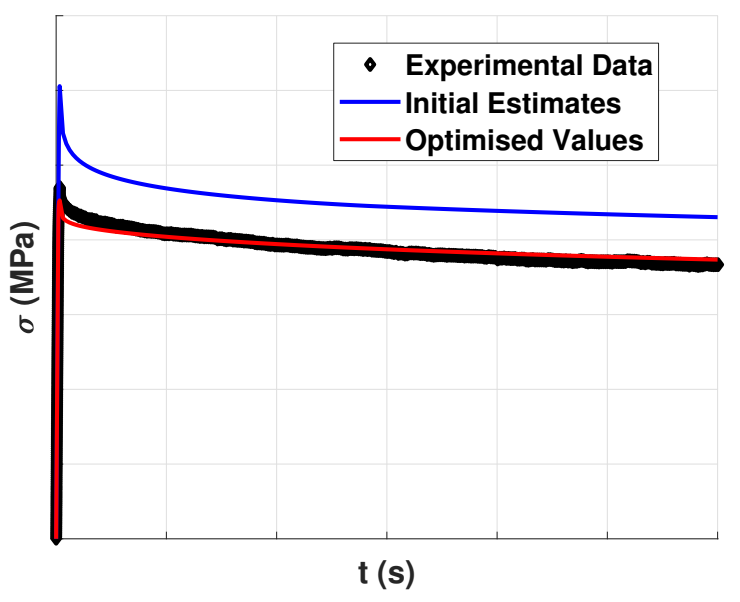

(c)

Figure 6: A summary of data used to estimate hardening law parameters for RR1000 at $750^{\circ} \mathrm{C}$, showing (a) Armstrong-Frederick kinematic hardening law application (assuming no time dependency) and optimised hardening parameters response (including time dependencies) plotted against (b) experimental $\sigma / \epsilon$ data and (c) experimental $\sigma / t$ data, taken from monotonic loadings. 


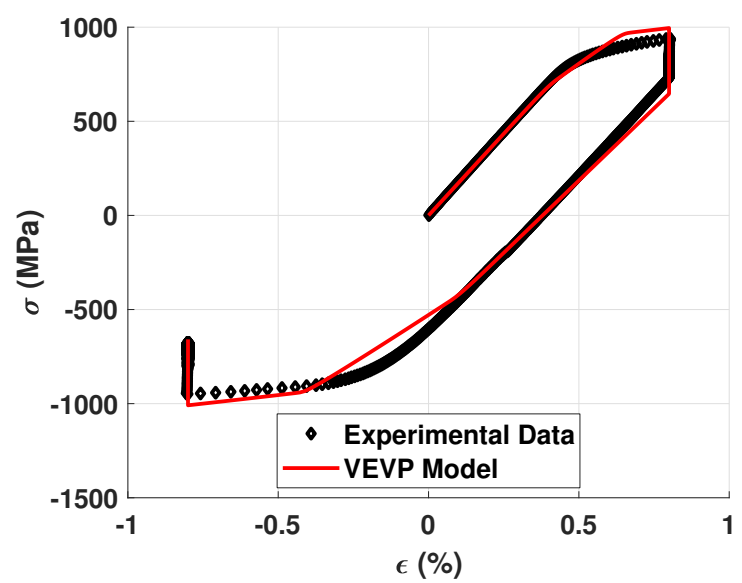

(a)

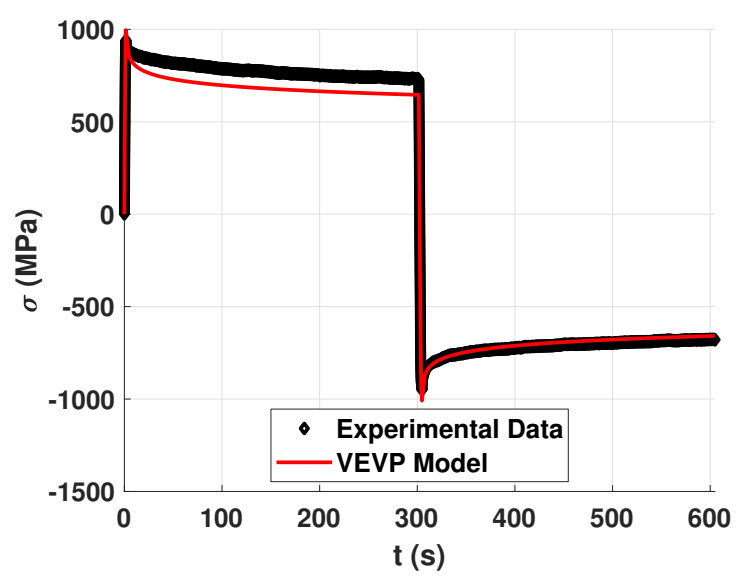

(b)

Figure 7: A comparison of cyclic experimental data $\left(750^{\circ} \mathrm{C}\right.$ isothermal conditions) and viscoelastic - viscoplastic model predictions for monotonic and first reversal loading cycles. Showing (a) $\sigma / \epsilon$ and (b) $\sigma / t$ behaviours to illustrate hardening and time dependent phenomena, respectively.

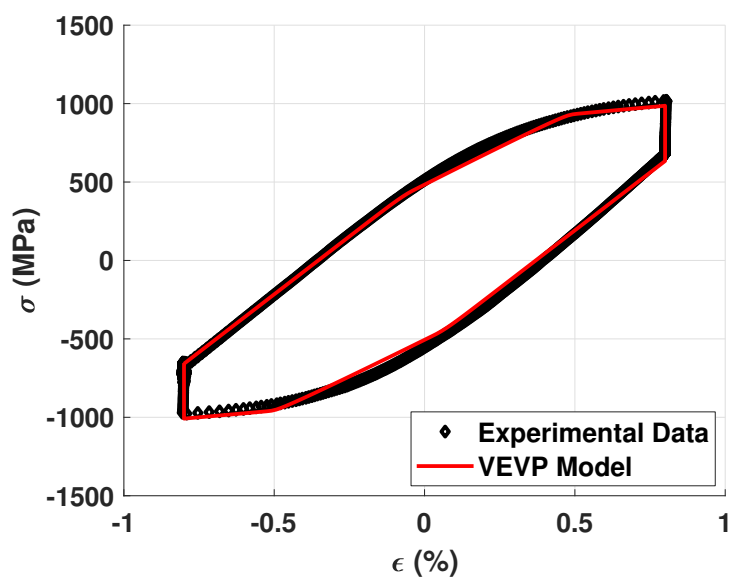

(a)

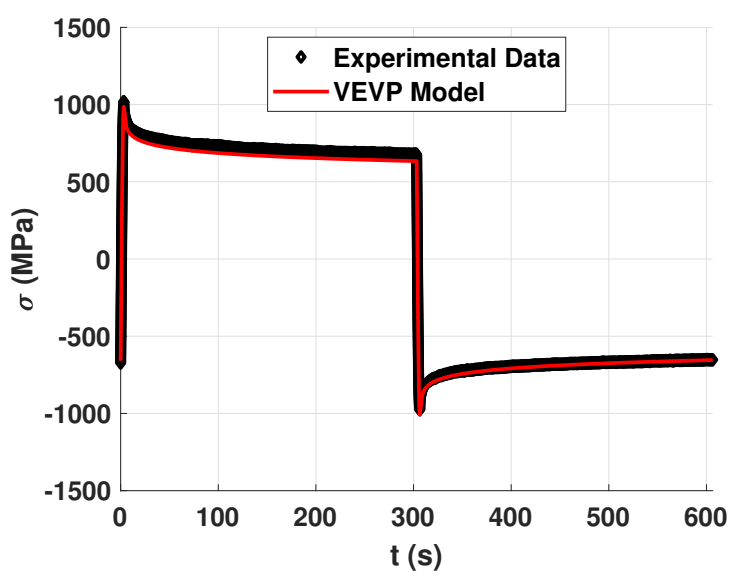

(b)

Figure 8: A comparison of cyclic experimental data $\left(750^{\circ} \mathrm{C}\right.$ isothermal conditions) and viscoelastic - viscoplastic model predictions for first full loading cycle (cycle 2). Showing (a) $\sigma / \epsilon$ and (b) $\sigma / t$ behaviours to illustrate hardening and time dependent phenomena, respectively. 


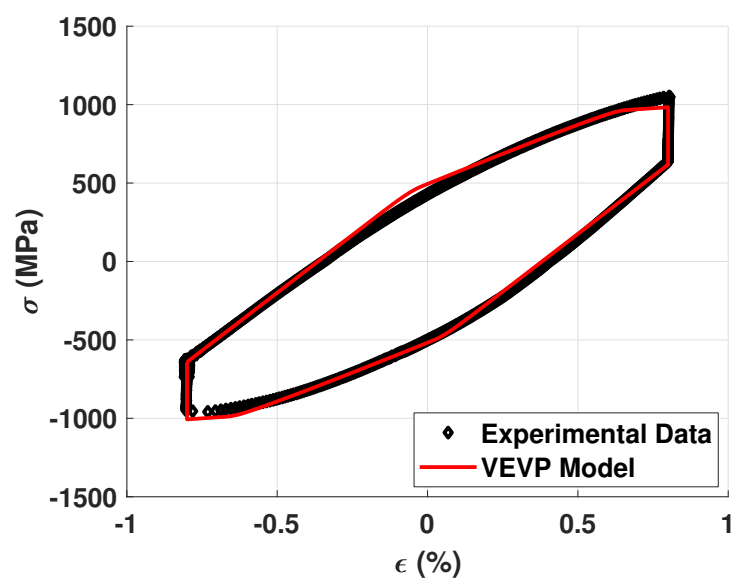

(a)

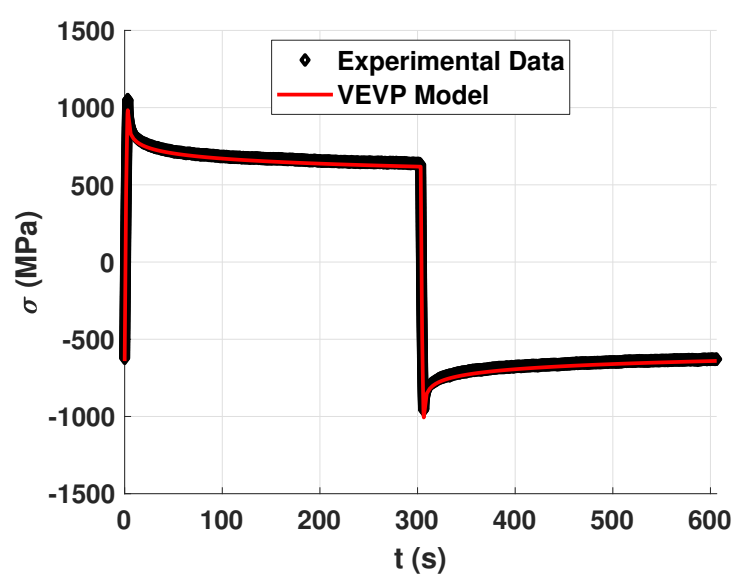

(b)

Figure 9: A comparison of cyclic experimental data $\left(750^{\circ} \mathrm{C}\right.$ isothermal conditions) and viscoelastic - viscoplastic model predictions for cycle 10 . Showing (a) $\sigma / \epsilon$ and (b) $\sigma / t$ behaviours to illustrate hardening and time dependent phenomena, respectively.

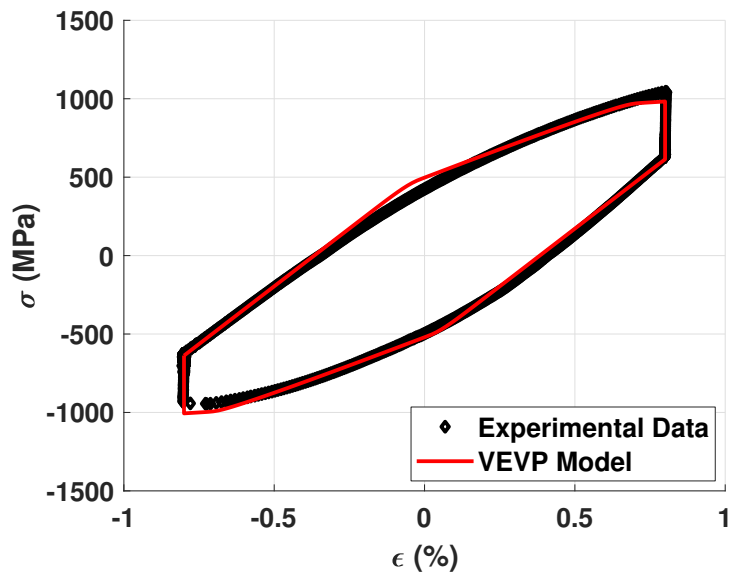

(a)

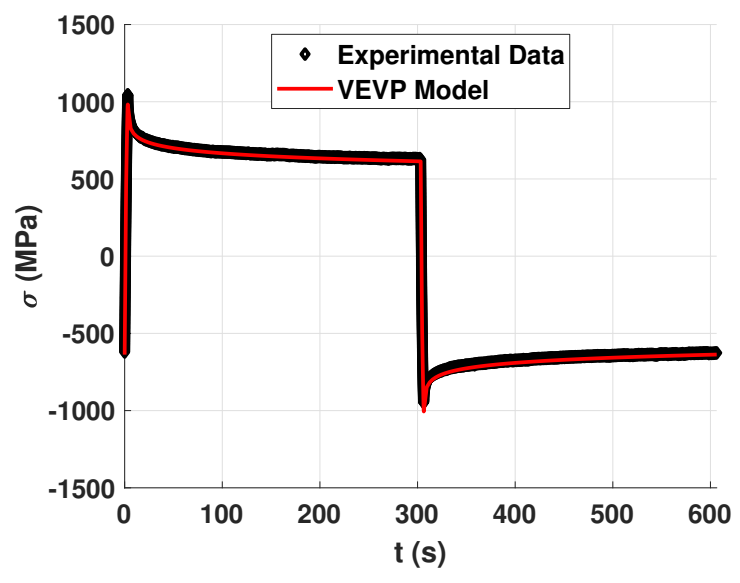

(b)

Figure 10: A comparison of cyclic experimental data $\left(750^{\circ} \mathrm{C}\right.$ isothermal conditions) and viscoelastic - viscoplastic model predictions for cycle 15 . Showing (a) $\sigma / \epsilon$ and (b) $\sigma / t$ behaviours to illustrate hardening and time dependent phenomena, respectively. 


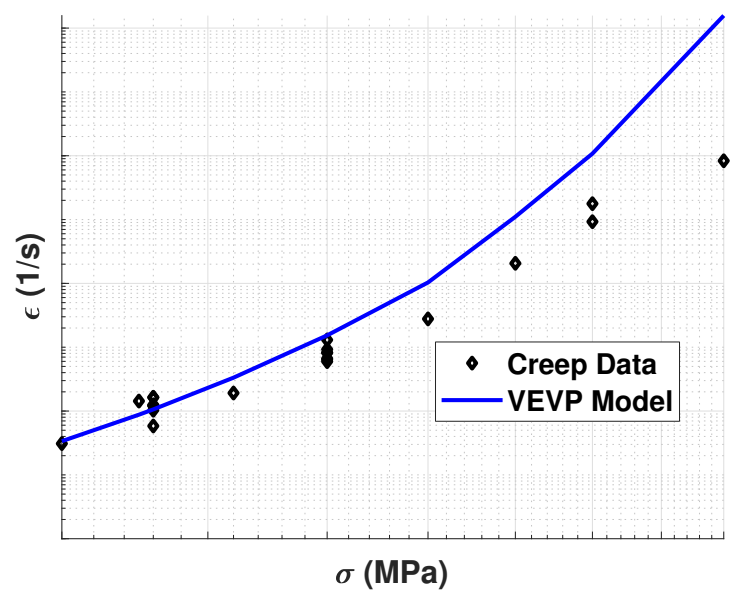

Figure 11: A comparison of strain rates predicted by the viscoelastic viscoplastic model and experimentally observed under creep conditions, determined for RR1000 at $750^{\circ} \mathrm{C}$.

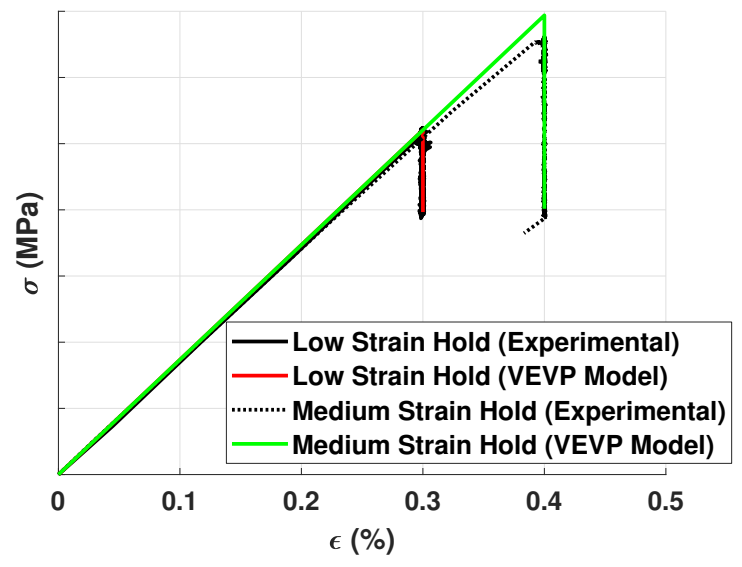

(a)

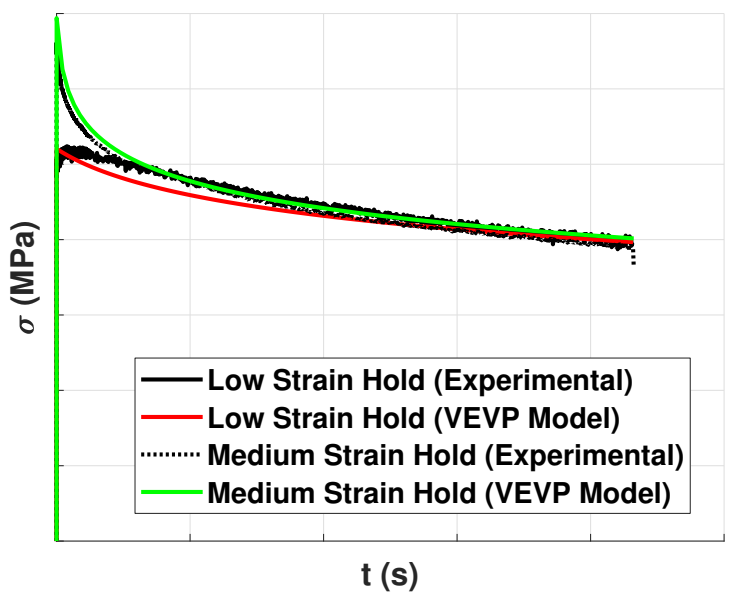

(b)

Figure 12: A comparison of experimental stress relaxation data $\left(750^{\circ} \mathrm{C}\right.$ isothermal conditions) and viscoelastic - viscoplastic model predictions for different holds strains. Showing (a) $\sigma / \epsilon$ and (b) $\sigma / t$ behaviours to illustrate hardening and time dependent phenomena, respectively.

\section{On the Inclusion of An-isothermal Effects}

A viscoelastic - viscoplastic model has been developed and calibrated against isothermal RR $1000750^{\circ} \mathrm{C}$ data. Comments will now be made on methods to extend the model to an-isothermal loading conditions using limited sets of data for material parameter determination as, after the definition of Helmholtz free energy (see equation (1)), this is relatively straightforward. At present however experimental data is too limited to provide any meaningful validation of the an-isothermal form of the viscoelastic-viscoplastic material model. So called "flight relevant" an-isothermal cycles have been applied to the RR1000 material in experimental programs, however load magnitudes are typically too low to induce any significant plasticity and load hold periods are too short for appreciable stress relaxation. These waveforms are primarily used in crack initiation investigations rather than for constitutive response evaluation and experience has shown that hysteresis "loops" (which are almost completely closed) can be adequately predicted by temperature 
dependent linear elastic material models. Isothermal calibration of the viscoelastic - viscoplastic material model is presented above and a brief explanation of how the model can be extended for an-isothermal conditions is reported here for the interested reader. Future work will look to validate an-isothermal predictions using large amplitude loading waveforms which incorporate a range of loading rates.

Temperature rate effects for a generic thermodynamic force $A$ (with associated flux $a$ ) can be determined by equation (23) [7]. By way of example, Armstrong-Frederick back stress functions may be extended to consider an-isothermal loadings by equation (24). It is clear that temperature dependent material parameters will need to be evaluated at instantaneous temperatures, however equation (24) illustrates that for some material parameters $\left(C_{i}^{\beta}\right.$, for example) an evaluation of the derivative of the related temperature dependent function will also be required for thermodynamic consistency. Several comments on the form of these temperature dependent material parameter functions may be made from observations made thus far. Firstly, the lack of stress relaxation in $400^{\circ} \mathrm{C}$ data (see figure 4) indicates that flow rule multipliers $\left(A^{V E]}\right.$ and $\left.A^{V P}\right)$ should tend towards 0 at this temperature. Furthermore, the lack of rate sensitivity suggests that $R_{o}^{V E}$ should tend towards $\sigma_{y}$ at $400^{\circ} \mathrm{C}$, such that the viscoelastic limit surface lies on top of the viscoplastic surface and deformation is entirely elastic prior to plasticity. Temperature dependencies in hardening parameters can of course be approximated by fitting the model to cyclic data at $400^{\circ} \mathrm{C}$ (see figure 13 by way of example). Intermediate viscoelastic parameters may be approximated by considering the correlation between viscoelastic strain rates and creep data, as demonstrated in figure 5 (b). Example viscoelastic flow rule fittings applied to creep data are shown in figure 14 (a) and (b) for $650^{\circ} \mathrm{C}$ and $700^{\circ} \mathrm{C}$ loading conditions, respectively.

$$
\begin{gathered}
\dot{A}=\frac{\partial^{2} \psi}{\partial a_{k} a_{j}} \dot{a}_{j}+\frac{\partial^{2} \psi}{\partial a_{k} T} \dot{T} \\
\dot{\chi}_{i}^{\beta}=\frac{2}{3} C_{i}^{\beta} \dot{\boldsymbol{\epsilon}}_{\beta}-\gamma_{i}^{\beta} \chi_{i}^{\beta} \dot{\lambda}_{\beta}+\frac{1}{C_{i}^{\beta}} \frac{\partial C_{i}^{\beta}}{\partial T} \dot{T}
\end{gathered}
$$

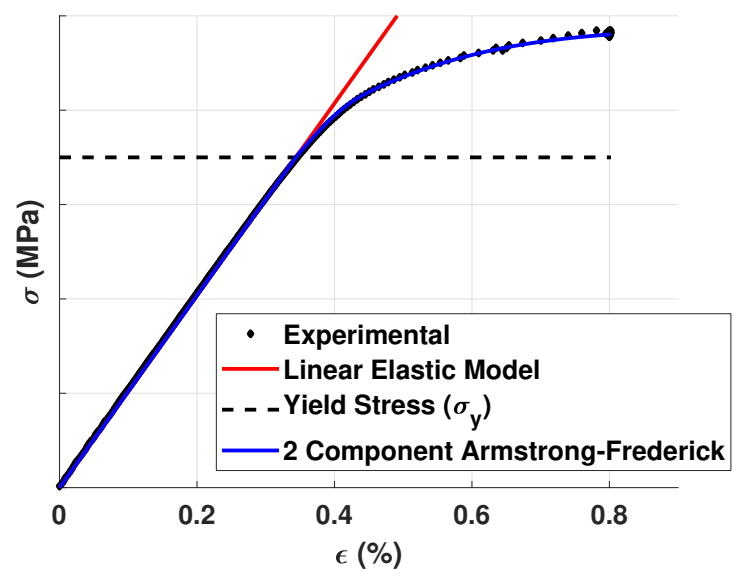

Figure 13: Armstrong-Frederick hardening laws fitted to R1000 $400^{\circ} \mathrm{C}$ data. Note that no time dependency is assumed. 


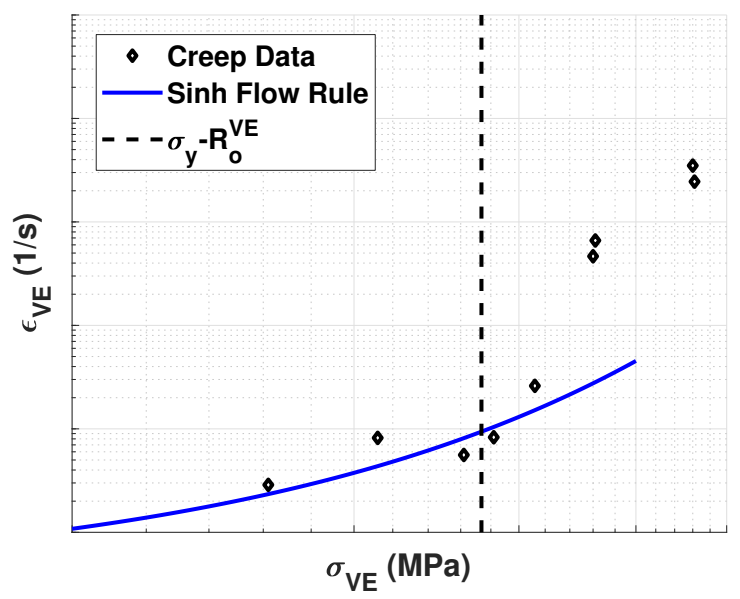

(a)

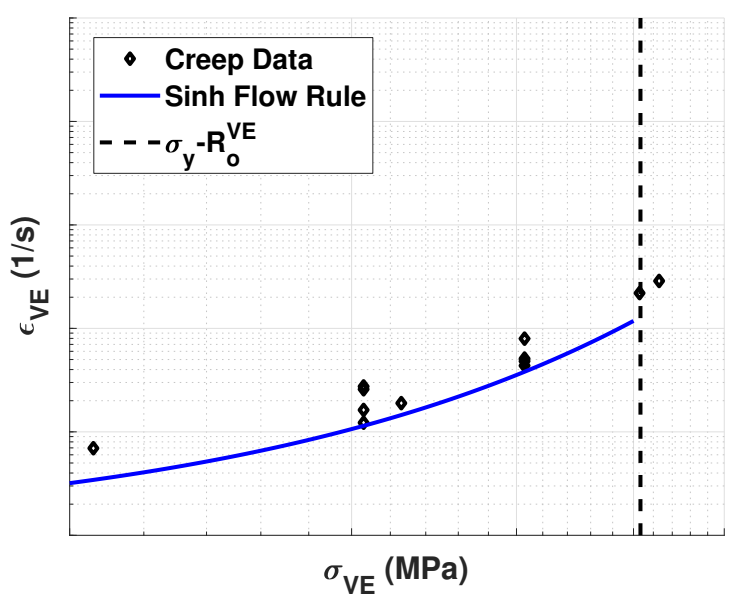

(b)

Figure 14: The estimation of viscoelastic flow rule parameters from minimum creep strain rates (found at constant stress), shown for RR1000 at (a) $650^{\circ} \mathrm{C}$ and (b) $700^{\circ} \mathrm{C}$.

\section{Discussion and Conclusions}

A thermodynamically based viscoelastic - viscoplastic material model has been developed here and calibrated for isothermal $750^{\circ} \mathrm{C}$ RR1000 material responses. The inclusion of Viscoelasticity allows stress relaxation at low loads to be approximated, which would not normally be possible for elastic - viscoplastic model formulations. The calibrated model (i.e. with optimised material parameters) shows good agreement with trapezoidal waveform cyclic experimental data. Stress relaxations at high and low load are generally well predicted and creep strain rate data shows good agreement with model estimates, suggesting that the double sinh flow rule formulation implemented here is capable of representing rate dependencies over a wide range of loading conditions.

The analysis of creep data, such as in figure 11 and figure 5 (b), offers a convenient way to calibrate flow rule terms in the absence of stress relaxation data. Considering creep data, however, also prompts interesting questions relating to how the model developed here would respond to typical creep loading waveforms (e.g. constant stress conditions). Assuming an applied stress is of sufficient magnitude to "activate" viscoelasticity related time dependent terms (i.e. it is greater than $R_{o}^{V E}$ ), $\epsilon_{V E}$ will accumulate. Under uniaxial loading $\epsilon_{V E}$ can be expressed as a scalar that is equivalent to so called creep strain. This equivalence is justified by noting that creep is a dissipative process observed for loads below yield and, in the viscoelastic - viscoplastic model, $\epsilon_{V E}$ relates to a dissipative process that is active under similar conditions. It can be seen, from the definition of the dissipation potential in equation (10), that the rate of accumulation of $\boldsymbol{\epsilon}_{V E}$ (denoted in the present work as $\dot{\boldsymbol{\epsilon}}_{V E}$ ) is dependent on the viscoelastic "overstress" $f^{V E}$. The value $f^{V E}$ will itself depend on the viscoelastic yield surface kinematic hardening parameter $\chi^{V E}$, see equation (6). As $\epsilon_{V E}$ accumulates under constant stress loading conditions $\chi^{V E}$ will evolve (see equation (14))and $\dot{\boldsymbol{\epsilon}}_{V E}$ will reduce. As time approaches infinity $f^{V E}$ will approach zero due to the increasing size of the viscoelastic limit locus, however the speed of this process will of course depend on the material parameters used in the associated flow rule. The reduction in $\dot{\boldsymbol{\epsilon}}_{V E}$ explained here for constant stress loading conditions offers a way in which primary creep effects can manifest in the model. Furthermore these observations indicate that primary creep regeneration effects, wherein creep strain rates are higher than expected after a load is reversed and then reapplied, could also manifest in the developed model. A certain amount of recovery in $\chi^{V E}$ will take place when loads are reversed, meaning that after reapplication of the load $\dot{\boldsymbol{\epsilon}}_{V E}$ magnitudes would be greater than those observed that just before load removal. Primary creep has not been explored in the present work due to a lack a relevant experimental data and the application of the simplifying assumptions in equation (16). Future work will look to address this omission, with particular 
emphasis placed on how primary creep data can be used to calibrate viscoelastic limit surface evolution parameters.

It is worth noting that the formulation presented here requires the definition of 15 material parameters. This is considerably less than the 22 used in other viscoelastic - viscoplastic models (for example, see Benaarbia et al. [18]). Much of the complexity in determining material parameters can be avoided if a flow rule is implemented rather than multiple rheological elements (for example, spring-damper Kelvin-Voigt elements). Hardening behaviour is generally well predicted in cyclic data, however some deviations in monotonic response behaviours is noted. Additional Armstrong-Frederick terms (or back stresses) or a refined objective function definition in optimisation algorithms may be required; future work will explore these and will look to add static recovery terms to kinematic hardening expressions. An-isothermal extensions to the formulation have been briefly discussed and methods to determine temperature dependent material parameter functions from limited experimental data to be examined. The definition of viscoelastic strain in the presented material model offers exciting opportunities for strain partitioning failure models, for example. Application of such lifing models with additional viscoelastic strain amplitude components will be investigated in future work.

\section{Acknowledgements}

This project has received funding from the European Union's Horizon 2020 research and innovation programme and Joint Undertaking Clean Sky 2 under grant agreement No 686600.

\section{References}

[1] Dieter G. Mechanical mettalurgy. 4th ed. McGraw Hill; 1988.

[2] Ince A, Glinka G. A generalized fatigue damage parameter for multiaxial fatigue life prediction under proportional and non-proportional loadings. International Journal of Fatigue. 2014;62:34-41.

[3] Chaboche JL, Rousselier G. On the plastic and viscoplastic constitutive-equations - part 1: Rules developed with internal variable concept. Journal of Pressure Vessel Technology - Transactions of the ASME. 1983;105:153-158.

[4] Chaboche JL, Rousselier G. On the plastic and viscoplastic constitutive-equations - part 2: Application of internal variable concepts to the 316 stainless-steel. Journal of Pressure Vessel Technology - Transactions of the ASME. 1983;105:159-164.

[5] Mroz Z. On the description of anisotropic work hardening. Journal of the Mechanics and Physics of Solids. 1967;15:163-175.

[6] Dafalias YF, Popov EP. Plastic internal variables formalism of cyclic plasticity. Journal of Applied Mechanics. 1976;43:645-651.

[7] Chaboche JL. A review of some plasticity and viscoplasticity constitutive theories. International Journal of Plasticity. 2008;24:1642-1693.

[8] Prager N. The theory of plasticity: A survey of recent achievements. Proceedings of the Institute of Mechanical Engineers. 1955;169:41-57.

[9] Ziegler H. A modification of Prager's hardening rule. Quarterly of Applied Mathematics. 1959;17:55-65.

[10] Armstrong PJ, Frederick CO. A mathematical representation of the multiaxial Bauschinger effect. Berkeley Nuclear Laboratories; 1966.

[11] Zhan Z, Fernando US, Ton J. Constitutive modelling of viscoplasticity in a nickel-based superalloyat high temperature. International Journal of Fatigue. 2008;30:1314-1323. 
[12] Cornet C, Zhao LG, Tong J. A study of cyclic behaviour of a nickel-based superalloy at elevated temperature using a viscoplastic-damage model. International Journal of Fatigue. 2011;33:241-249.

[13] Zhao LG, Tong J. A viscoplastic study of crack-tip deformation and crack growth in a nickel-based superalloy at elevated temperature. Journal of the Mechanics and Physics of Solids. 2008;56:3363-3378.

[14] Kirka MM, Neu RW. Crystal viscoplasticity of a Ni-base superalloy in the aged state. Journal of Engineering Materials and Science. 2019;141:011003-1-011003-12.

[15] Zhang X, Oskay C. Polycrystal plasticity modeling of nickel-based superalloy IN 617 subjected to cyclic loading at high temperature. Modelling and Simulation in Materials Science and Engineering. 2016;24.

[16] Yu C, Kang G, Chen K, Lu F. A thermo-mechanically coupled nonlinear viscoelastic-viscoplastic cyclic constitutive model for polymeric materials. Mechanics of Materials. 2017;105:1-15.

[17] Launay A, Maitournam MH, Marco Y, Raoult I, Szmytka F. Cyclic behaviour of short glass fibre reinforced polyamide: Experimental study and constitutive equations. International Journal of Plasticity. 2011;27:1267-1293.

[18] Benaarbia A, Rouse JP, Sun W. A thermodynamically-based viscoelastic-viscoplastic model for the high temperature cyclic behaviour of 9-12\% Cr steels. International Journal of Plasticity. 2018;107:100-121.

[19] Cai X, Steinmann P, Yao X, Wang J. Thermodynamic formulation of a unified multi-mechanism continuum viscoplastic damage model with application to high-Cr steels. International Journal of Plasticity. 2019;114:15-39.

[20] Wang J, Steinmann P, Rudolph J, Willuweit A. Simulation of creep and cyclic viscoplastic strains in high-Cr steel components based on a modified Becker-Hackenberg model. International Journal of Pressure Vessels and Piping. 2015;128:36-47.

[21] Chaboche JL, Lemaitre J. Mechanics of solid materials. Cambridge; 1985.

[22] Cottrell AH. Dislocations and plastic flow in crystals. 1st ed. Oxford, U.K.: Oxford University Press; 1953. 